\title{
Double knockouts in human embryonic stem cells
}

\author{
Jizhong Zou ${ }^{1,2}$, Rory Cochran ${ }^{3}$, Linzhao Cheng ${ }^{1,2,3}$ \\ ${ }^{I}$ Stem Cell Program, Institute for Cell Engineering, Johns Hopkins University School of Medicine, Baltimore, MD 21205, USA; ${ }^{2} D i-$ \\ vision of Hematology, Department of Medicine, Johns Hopkins University School of Medicine, Baltimore, MD 21205, USA; ${ }^{3}$ Gradu- \\ ate Program in Cellular and Molecular Medicine, Johns Hopkins University School of Medicine, Baltimore, MD 21205, USA \\ Cell Research (2010) 20:250-252. doi: 10.1038/cr.2010.29; published online 1 March 2010
}

Gene targeting is the method of precise genome modification that utilizes the innate DNA repair mechanism in cells, known as homologous recombination. At its genesis, gene targeting in mouse embryonic stem (ES) cells was a breakthrough for genetics and developmental biology. Its application has enhanced researchers' ability to study gene function and model human disease, which was recognized by the Nobel Prize in Physiology or Medicine 2007.

Although this immensely powerful technology is versatile to achieve gene knockouts (targeted deletion of endogenous gene), or knockins (targeted insertion of exogenous DNA) in mice, gene targeting has been extremely challenging in human ES cells due to their much lower colony forming efficiency. Since the first report by Zwaka and Thomson in 2003 [1], only about a dozen papers showed successful gene targeting of one allele in human ES cells (see a recent review [2]). Considering that most genes are on non-sex chromosomes that exist in two copies, a double knockout strategy is highly desirable. A report by $\mathrm{Bu}$ et al., in this issue [3], and a recent paper in Cell Stem Cell [4], demonstrated that in human ES cells not only can a double

Correspondence: Linzhao Cheng

Tel: 410-614-6958; Fax: 443-287-5611

E-mail: 1cheng@welch.jhu.edu knockout be achieved but it also can be conditional or inducible to allow more control in functional studies.

In the article by $\mathrm{Bu}$ et al. [3], the authors extended their previous strategy of the mouse BAF250a (ARID1A) double knockout into human ES cells. In the initial step, the first allele was targeted to temporally incorporate a $F R T$-flanked selection cassette, which was later excised by the expression of yeast-derived Flippase (Flp) to convert this allele back to wild-type while leaving a loxP-flanked exon nearby. The reverted wild-type allele is made in the process to ensure that functional protein will be present when the second allele is disrupted in the event the gene is indispensable for human ES cell survival or growth. Next, the second allele was conveniently targeted using the same donor vector, resulting in loxP-flanked exons on both alleles. Finally, Cremediated deletion of the loxP-flanked exons created the double knockout human ES cells (Figure 1A). This "two birds with one stone" strategy simplifies the vector construction as well as the detection methods for gene-targeted clones. In addition, using one type of drug makes it easier to determine the right selection concentration because it can be influenced by the expression level of the drug-resistant gene and thereby needs to be tested experimentally. One important observation is that the gene targeting of the second allele using the same donor vector appeared to be less favorable, as only $10 \%$ of clones have the correct biallelic modification. This could be due to the donor DNA having slightly higher homology with the reverted first allele than with the unmodified second allele.

In another recent report, a group used the bacterial artificial chromosome (BAC) to achieve a double knockout of the ATM or TP53 gene in human ES cells [4]. BAC vectors take advantage of long homology arms to compensate for the low gene targeting efficiency in human ES cells. Two targeting vectors with different drug-resistant genes were used to select double knockout clones for either the ATM or TP53 gene. Although technically challenging construction of large BAC vectors can now be facilitated by recombineering technology, verification of the targeted integration and exclusion of random integrations are more difficult than using smaller DNA segments delivered by a plasmid. While these recent double knockout reports used different donor design strategies, they both targeted genes that are actively expressed in undifferentiated human ES cells. It will be interesting to see if these two strategies can also be applied in double knockouts of transcriptionally inactive genes that are turned later at various developmental stages and often play key roles in human biology and disease. The gene targeting efficiencies of silent 
A
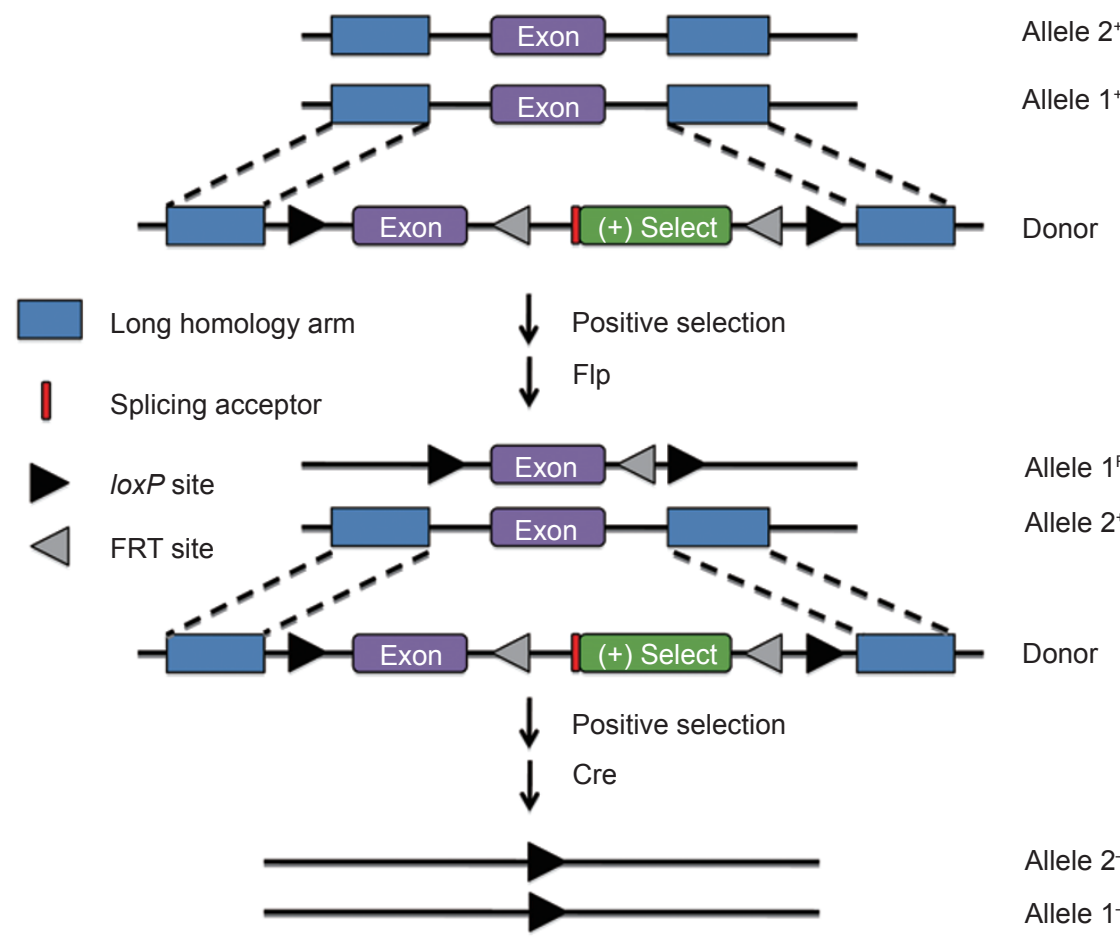

Efficiency $<10^{-6}$

B

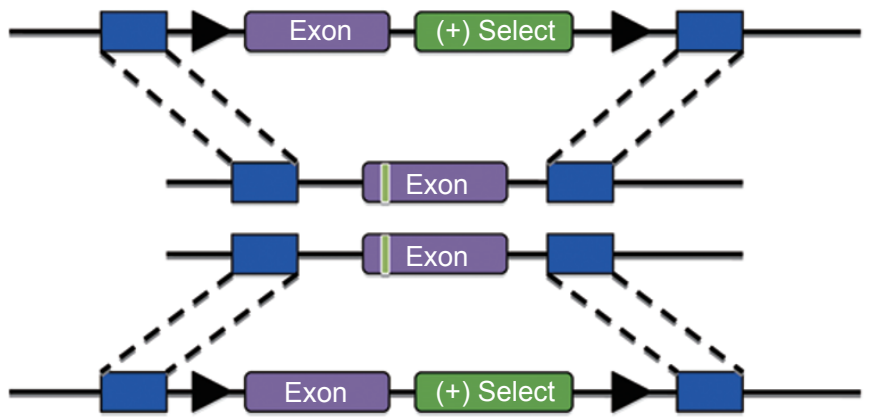

Donor

Allele $1^{+}$

Allele $2^{+}$

Donor

Short homology arm

Positive selection

ZFN cut site

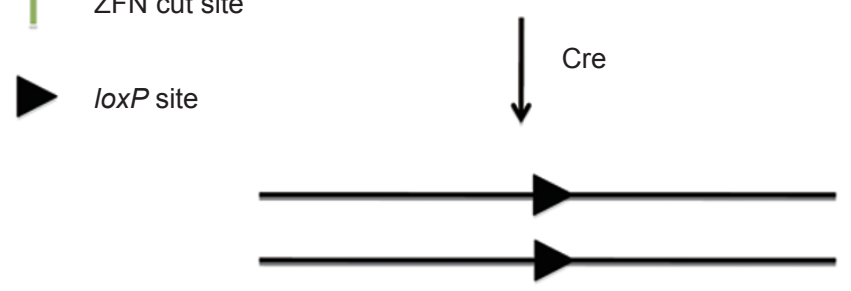

Efficiency $\sim 10^{-3}$ genes in undifferentiated human ES cells are dramatically lower due to the epigenetic conditions and chromosomal microenvironment [2].

Additional gene targeting strategies are being developed to attain highefficiency in both human ES cells and newly established induced pluripotent stem (iPS) cells. One strategy utilizes a novel technology called zinc-finger nuclease (ZFN), which is designed to fuse multiple zinc-finger DNA binding domains to a non-specific FokI nuclease domain. Upon dimer formation, two ZFNs can create a sequence-specific DNA double strand break (DSB) to stimulate homologous recombination [5]. Recently, this strategy has been employed to successfully target both expressed and silent genes in human ES and iPS cells $[6,7]$. The gene targeting efficiency can be enhanced by $>100-1$ 000 -fold when $\mathrm{ZFN}$-expressing vectors are co-delivered with donor vector,

Figure 1 Schematic of conditional double knockout strategies. (A) Strategy of conditional double knockouts used by $\mathrm{Bu}$ et al. [3] A donor DNA vector contains targeted exon flanked by loxP sites and a $F R T$-flanked positive selection cassette $[(+)$ Select)] to achieve reverted wild-type allele $1^{\text {Rev }}$ at the initial step. After $(+)$ selection of targeted clones and excision of the selection cassette by Flp that removes DNA between FRT sites, the same donor vector is used to target the allele 2 . After the same (+) selection and FIp-FRT excision, reverted exons on both alleles will be excised by Cre-mediated recombination between two loxP sites. The typical efficiency for each homologous recombination step is low $\left(\sim 10^{-6}\right)$ in mammalian cells and long homology arms are often required. (B) Enhancing gene targeting by zinc finger nucleases (ZFNs) that make a double strand break (DSB) at the targeted exon. ZFNs are shown to dramatically enhance homologous recombination efficiency by $\sim 1000$-fold. Two alleles could be simultaneously targeted in the same cells when facilitated by ZFNs. Shorter homology arms are often sufficient in ZFN-mediated gene targeting. 
reaching $100 \%$ for active genes and $\sim 10 \%$ for silent genes after a positive selection. An additional advantage to using $\mathrm{ZFN}$-mediated gene targeting is that it requires fairly short homology arms, typically 1-2 kb (Figure 1B). Homozygously targeted human ES and iPS cells were obtained with high efficiency [7]. Although this method is powerful and auspicious, it relies on design and screening of highly specific and active ZFNs to achieve efficient and precise gene targeting. Consequently, better screening for off-targets of ZFNs needs to be pursued before clinical applications of selected human ES or iPS clones after gene targeting.

Over the past three years, there has been a wave of burgeoning technology to achieve gene targeting in human pluripotent stem cells. In addition to the non-viral vector systems mentioned above, non-integrating lentivirus [8], adenovirus [9] and adenovirusassociated virus [10] all have been used in human ES cell gene targeting. Inactivating an endogenous gene by knockout or inserting a reporter gene by knockin in human ES cells enables researchers to study human develop- ment and disease in human cell-based systems that differ from mouse. The importance of using human cell-based systems is underscored by the recent double knockout in human ES cells, showing contrasting human phenotypes from their mouse counterparts $[3,4]$. Not only can patient-specific human iPS cells reprogrammed from somatic cells be used to model diseases with inherited or acquired genetic mutations, but they also show promise for stem cell-based gene therapy in regenerative medicine for many monogenic disorders.

\section{References}

1 Zwaka TP, Thomson JA. Homologous recombination in human embryonic stem cells. Nat Biotechnol 2003; 21:319-321.

2 Giudice A, Trounson A. Genetic modification of human embryonic stem cells for derivation of target cells. Cell Stem Cell 2008; 2:422-433.

3 Bu L, Gao X, Jiang X, Chien KR, Wang $\mathrm{Z}$. Targeted conditional gene knockout in human embryonic stem cells. Cell Res 2010; 20:379-382

4 Song H, Chung SK, Xu Y. Modeling disease in human ESCs using an effi- cient BAC-based homologous recombination system. Cell Stem Cell 2010; 6:80-89.

5 Carroll D. Progress and prospects: Zinc-finger nucleases as gene therapy agents. Gene Ther 2008; 15:1463-68.

6 Zou J, Maeder ML, Mali P, et al. Gene targeting of a disease-related gene in human induced pluripotent stem and embryonic stem cells. Cell Stem Cell 2009; 5:97-110.

7 Hockemeyer D, Soldner F, Beard C, et $a l$. Efficient targeting of expressed and silent genes in human ESCs and iPSCs using zinc-finger nucleases. Nat Biotechnol 2009; 27:851-857.

8 Lombardo A, Genovese P, Beausejour $\mathrm{CM}$, et al. Gene editing in human stem cells using zinc finger nucleases and integrase-defective lentiviral vector delivery. Nat Biotechnol 2007; 25:1298-1306.

9 Suzuki K, Mitsui K, Aizawa E, et al. Highly efficient transient gene expression and gene targeting in primate embryonic stem cells with helper-dependent adenoviral vectors. Proc Natl Acad Sci USA 2008; 105:13781-13786.

10 Mitsui K, Suzuki K, Aizawa E, et al. Gene targeting in human pluripotent stem cells with adeno-associated virus vectors. Biochem Biophys Res Commun 2009; 388:711-717. 